\title{
Light-Actuated Liquid Crystal Elastomer Prepared by Projection Display
}

\author{
Juan Chen, Oluwafemi Isaac Akomolafe, Jinghua Jiang * and Chenhui Peng *
}

check for updates

Citation: Chen, J.; Akomolafe, O.I.;

Jiang, J.; Peng, C. Light-Actuated

Liquid Crystal Elastomer Prepared by Projection Display. Materials 2021, 14 , 7245. https://doi.org/10.3390/ ma14237245

Academic Editors: Uroš Tkalec and Dong Ki Yoon

Received: 8 November 2021

Accepted: 24 November 2021

Published: 27 November 2021

Publisher's Note: MDPI stays neutral with regard to jurisdictional claims in published maps and institutional affiliations.

Copyright: (c) 2021 by the authors. Licensee MDPI, Basel, Switzerland. This article is an open access article distributed under the terms and conditions of the Creative Commons Attribution (CC BY) license (https:// creativecommons.org/licenses/by/ $4.0 /)$.
Department of Physics and Materials Science, The University of Memphis, Memphis, TN 38152, USA; jchen12@memphis.edu (J.C.); oluwafemi.akomolafe@memphis.edu (O.I.A.)

* Correspondence: jjiang4@memphis.edu (J.J.); cpeng@memphis.edu (C.P.)

\begin{abstract}
Soft materials with programmability have been widely used in drug delivery, tissue engineering, artificial muscles, biosensors, and related biomedical engineering applications. Liquid crystal elastomers (LCEs) can easily morph into three-dimensional (3D) shapes by external stimuli such as light, heat, and humidity. In order to program two-dimensional (2D) LCE sheets into desired 3D morphologies, it is critical to precisely control the molecular orientations in LCE. In this work, we propose a simple photopatterning method based on a maskless projection display system to create spatially varying molecular orientations in LCE films. By designing different synchronized rotations of the polarizer and projected images, diverse configurations ranging from individual to 2D lattice of topological defects are fabricated. The proposed technique significantly simplified the photopatterning procedure without using fabricated masks or waveplates. Shape transformations such as a cone and a truncated square pyramid, and functionality mimicking the responsive Mimosa Pudica are demonstrated in the fabricated LCE films. The programmable LCE morphing behaviors demonstrated in this work will open opportunities in soft robotics and smart functional devices.
\end{abstract}

Keywords: photopatterning; director field; liquid crystal elastomers; light-driven

\section{Introduction}

Soft active materials with programmable shape changes have shown applications in drug delivery [1], artificial muscles [2,3], bioelectronics [4,5], and biomedicine [6]. Precise control of the directionality and magnitude of mechanical deformation is vital to program the shape-morphing behaviors. As a type of ordered crosslinked polymer, liquid crystal elastomers (LCEs) have shown remarkable structural transformations by patterning the complex molecular orientations [7]. The change in the molecular ordering of LCE under external stimuli such as light [8], heat [9], or humidity [10] gives rise to a variety of threedimensional (3D) shape morphs thanks to its anisotropic elasticity [11-13]. For example, Ware et al. demonstrated arrays of cones and anti-cones transformed from a flat LCE film by pixelating its two-dimensional (2D) director field [14]. Aharoni et al. morphed the arbitrary shape from 2D to 3D in thin LCE sheets via the inverse-design principle, where the shape is programmed by controlling the molecular orientations of the liquid crystal (LC) monomers [15]. Hence, encoding director orientations in LCE to program the shape-morphing remains non-trivial for developing previously non-achievable mechanical deformations and functionalities.

In order to produce LC with spatially-varying orientations, various techniques have been developed to pattern the 2D director field $[16,17]$. Rosenblatt's group created arbitrary arrays of 2D topological defects by using the nano-rubbing of atomic force microscope (AFM) probes [18,19]. The LC director field can also be patterned by using 2D channels micro-fabricated by photolithography $[15,20]$ or two-photon polymerization [21]. Recent advances in 3D printing have also enabled complex 3D shape transformations in LCE, but the alignment has to be realized by optimizing the printing path and material viscosity [22]. As such, both the nano-rubbing and 3D fabrication involve complicated processes and 
costly equipment, which will prolong the fabrication time and offer limited access for beginners [13]. The most commonly used technique in both industry and academia is the so-called photopatterning technique by using light to control the LC director field [23]. To imprint the directional alignment on the surface that the LC molecules will be interfacing with, a photosensitive azo-dye layer is coated on the surface [24]. By shining linearly polarized light on the azo-dye, the dye molecules will be aligned perpendicularly to the linear polarization direction through isomerization [23]. This alignment of azo-dye will be further used to control the orientation of LC molecules that follow the azo-dye orientations. In order to fabricate complex patterning of LC director field, light patterns with spatially-varying linear polarization are created by using the digital micro-mirror device [25], plasmonic metamasks [26,27], waveplates $[28,29]$, or specially-fabricated photomasks $[9,30]$. These optical elements are only designed to fabricate specific patterns, and if a new pattern is needed, a new device has to be fabricated, which will increase the production cost. If these optical elements are not used, a laser beam with pixel-by-pixel polarization has to be designed to create pixel-by-pixel orientations, which is time-consuming and costly [14]. Thus, an easily operating photopatterning technique without using fabricated masks or waveplates is highly desired.

In this work, we propose a maskless technique based on a projection display system to pattern complex molecular ordering in LCE film. The system is composed of a projector, a rotational linear polarizer, and focusing lenses. By synchronizing the rotation between the projected images and the polarizer, a variety of patterns with spatially varying director fields are created, which include individual topological defects and a 2D lattice of topological structures. The created patterns can be reconfigured by simply programming the designs of projected images and the rotations of the polarizer. When light-actuated LCE sheets are encoded with pre-designed ordered structures, 3D shape changes such as a cone and a truncated square pyramid are created via light irradiation. Based on the obtained shape-shifting and by cutting the LCE with certain geometries, a light-responsive bioinspired functionality similar to the response of Mimosa Pudica to the external stimuli is demonstrated. The demonstrated programmability of LCE shape changes by using the maskless photopatterning technique can be integrated with origami (Japanese art of paper folding) or kirigami (Japanese art of paper cutting) designs in the future, enabling further applications in soft robotics and smart materials.

\section{Experiment Results}

\subsection{Sample Preparation}

Glass slides are washed with detergent in an ultrasonic bath for $8 \mathrm{~min}$, then rinsed with deionized water, acetone, and isopropanol in sequence. To remove the excess liquid, the substrates are baked in an oven at $90^{\circ} \mathrm{C}$ for $10-15 \mathrm{~min}$. Afterward, they undergo a five-minute ultraviolet (UV) ozone treatment for deep cleaning. Homeotropic alignments are obtained by spin-coating polyimide PI-1211 on a pre-cleaned substrate at $3000 \mathrm{rpm}$ for $30 \mathrm{~s}$. This substrate is baked on a hot plate at $95^{\circ} \mathrm{C}$ for $2 \mathrm{~min}$, followed by one-hour baking at $180^{\circ} \mathrm{C}$.

The photopatterned substrate is prepared by spin-coating a layer of photosensitive azo-dye SD1 (DIC INC, Tokyo, Japan) solution on the surface at $3000 \mathrm{rpm}$ for $30 \mathrm{~s}$. The SD1 solution consists of $0.2 \mathrm{wt} \%$ SD1, Figure $1 \mathrm{c}$, and $99.8 \mathrm{wt} \% \mathrm{~N}, \mathrm{~N}$-dimethylformamide (DMF). The coated substrate is then subjected to 15 -min baking on the hot stage at $120^{\circ} \mathrm{C}$. The molecules of this azo-dye layer will be oriented perpendicular to the linearly polarized light on the photoalignment layer. 
a<smiles>C=CC(=O)OCCCCCCOc1ccc(C(=O)Oc2ccc(OC)cc2)cc1</smiles>

\section{LC monomer}

$\mathrm{b}$<smiles>C=CC(=O)OCCCOc1ccc(C(=O)Oc2ccc(OC(=O)c3ccc(OCCCOC(=O)C=C)cc3)c(C)c2)cc1</smiles><smiles>NC(=O)c1cc(N=Nc2ccc(-c3ccc(N=Nc4ccc(O)c(C(=O)O)c4)cc3[N+](=O)[O-])c([N+](=O)[O-])c2)ccc1O</smiles>

$\mathrm{d}$<smiles>O=C(c1ccccc1)C(O)(O)c1ccccc1</smiles>

Photo-initiator<smiles>C=CC(=O)OCCN(CC)c1ccc(/N=N/c2ccc([N+](=O)[O-])cc2)cc1</smiles>

DR1A

Figure 1. Chemical structures of azo dye and components of LCE. (a) LC monomer; (b)LC crosslinker; (c) Azo dye SD1; (d) Photo-initiator; (e) Disperse Red 1 acrylate (DR1A).

\subsection{Photopatterned Substrates Preparation}

The proposed maskless projection display setup is shown in Figure 2a. The setup is made of a projector (Epson, Suwa, Japan), two convex lenses, a controllable rotating polarizer, and a screen. The projector, with a wavelength of $300-700 \mathrm{~nm}$, has a resolution of $1920 \times 1080$ pixels and projects predesigned segments. Lens1 (LH-2 lens, Thorlabs, Newton, NJ, United States) and lens2 (convex lens, Thorlabs) are used to get the best focus and size of the projected pattern. A linear polarizer is attached to a motorized rotation stage (PRM1Z8, Thorlabs), connected to a brushed DC servo motor controller (Thorlabs). Thus, the angular speed of the polarizer can be precisely controlled by a LabView program. An SD1 substrate is placed on the screen and irradiated by the projected segments with linearly polarized light. The desired director field will be created on the substrate by the synchronized rotation of the polarizer and projection segments.

A director field is designed as $\hat{\mathbf{n}}=\left(n_{x}, n_{y}\right)=(\cos \theta, \sin \theta)$, and $\theta(x, y)=m \tan ^{-1} \frac{y}{x}+$ $\theta_{0}$, where $m$ is the topological charge, and $\theta_{0}$ indicates the distortion type. The desired director filed is divided into 36 pieces of $10^{\circ}$-segments evenly, Figure $2 \mathrm{~b}$. In order to get the best contrast, the segments are designed as white triangles on a black background, Figure $2 b$,c. These segments are arranged in 36 PowerPoint slides, respectively, which are further projected in sequence on the SD1 substrate with a time interval. The topological charge is defined as $m=R_{1} / R_{2}$, where $R_{1}$ and $R_{2}$ are the angular speeds of the polarizer and segments, respectively. The starting segment is always set along the $x$-axis, and the 36 segments rotate in a clockwise (CW) sense with a time interval of $10 \mathrm{~s}$. Note that an exposure time of $10 \mathrm{~s}$ is enough to imprint the alignment in the SD1 layer. As such, the SD1 substrate is irradiated with rotational segments at an angular speed $R_{2}=10^{\circ} / 10 \mathrm{~s}$. By programming the speed, starting position, and rotation direction of the polarizer, different director fields will be obtained. 

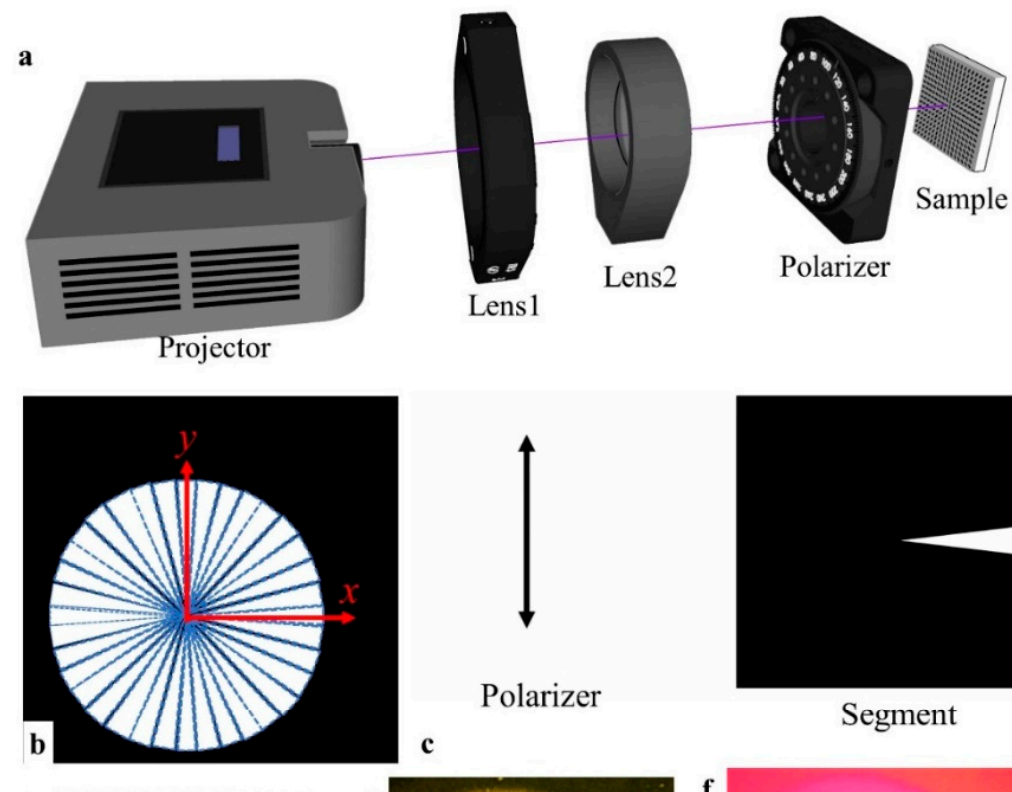

Polarizer
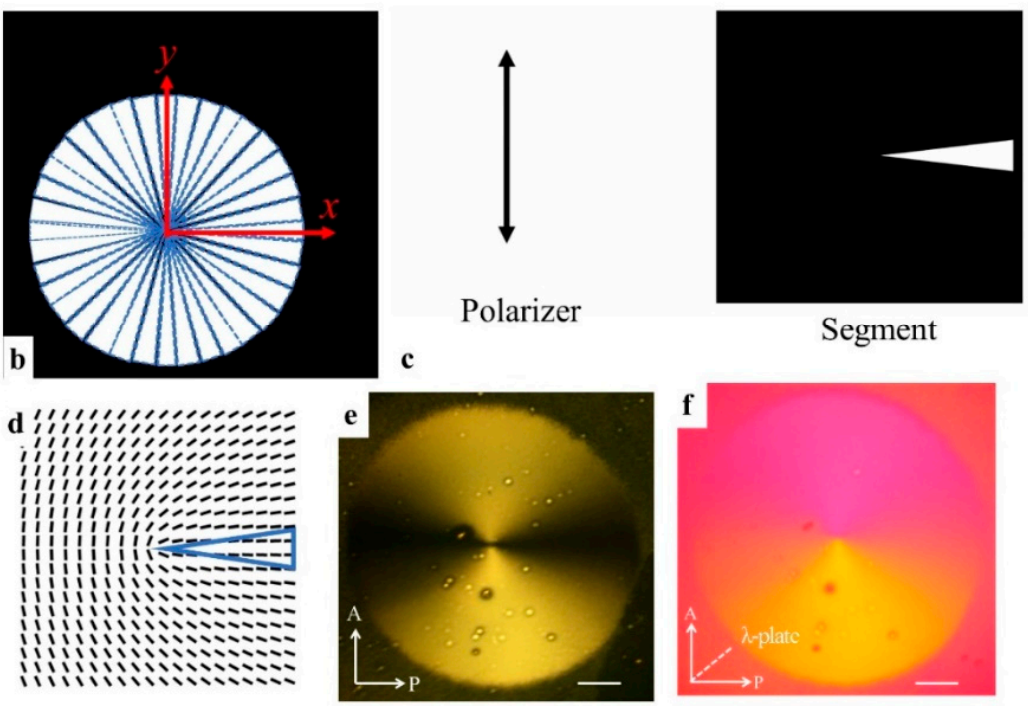

Segment
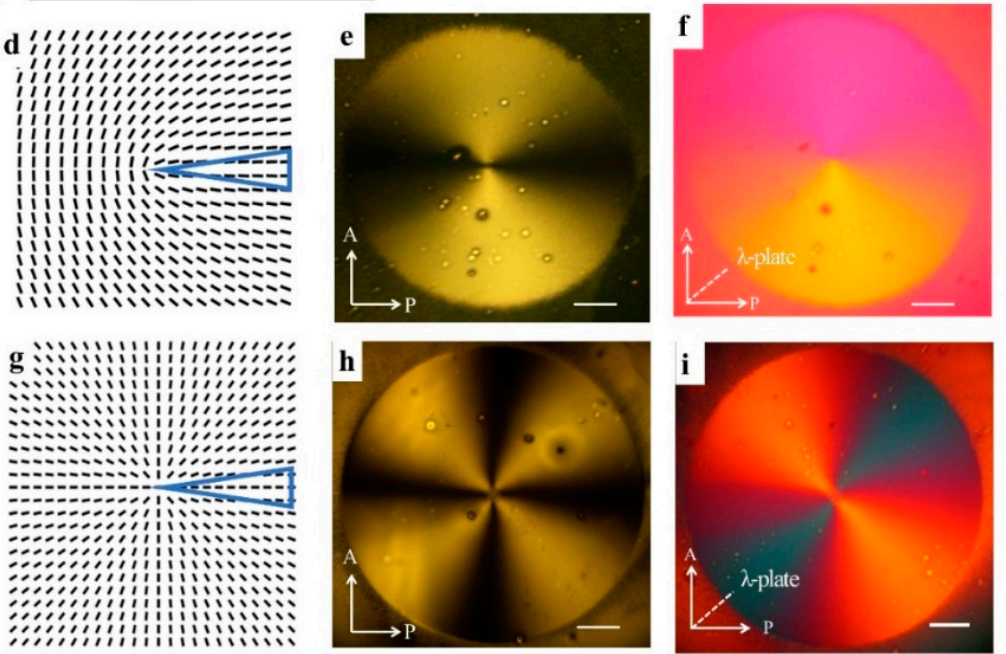

Figure 2. Illustration of photopatterning process. (a) A maskless photopatterning setup by projection display. (b) The pattern is divided into 36 segments evenly. (c) The starting positions of the polarizer and segments for producing director filed in $(\mathbf{d}, \mathbf{g})$. (d) The director field of $+1 / 2$ defect. (e,f) The POM images of $+1 / 2$ defect without (e) and with (f) the red plate. If the slow axis of the "red plate" is in the same direction as the LC alignment, the interference color will be "violet" or "blue", indicating an increase in retardance. (g) The director field for a radial +1 defect. (h,i) The POM images of the radial +1 defect without $(\mathbf{h})$ and with (i) the red plate. Scale bars in all images are $2.5 \mathrm{~mm}$. The blue triangles in $(\mathbf{d}, \mathbf{g})$ are the starting positions of the segments. P and A represent polarizer and analyzer.

For example, when the polarizer axis is aligned along the $y$-axis at the beginning of the process, Figure $2 \mathrm{c}$, and it jogs $5^{\circ}$ per $10 \mathrm{~s}$ in the CW sense, the angular speed of the polarizer is $R_{1}=5^{\circ} / 10 \mathrm{~s}$. The obtained topological charge will be $m=R_{1} / R_{2}=+1 / 2$, Figure $2 \mathrm{~d}$. The desired pattern with $+1 / 2$ defect will be created after a full $2 \pi$ rotation of segments, Figure $2 \mathrm{e}, \mathrm{f}$. For the director field of $m=+1$ with pure splay $\theta_{0}=0$, Figure $2 \mathrm{~g}$, the polarizer is programmed by LabView to start along the $y$-axis and maintain a speed of $R_{1}=10^{\circ} / 10 \mathrm{~s}$ in the CW sense. Hence, the obtained topological charge is $m=R_{1} / R_{2}=+1$. After a full rotation of segments, polarizing optical microscope (POM) images of the radial pattern with +1 defect, are shown in Figure $2 \mathrm{~h}$,i.

Therefore, by varying the speed, starting position, and rotation directions of the polarizer, the topological charge and distortion type can be well-tuned. A variety of half or integer topological defects can be produced without any fabricated masks or waveplates. The capability to create arbitrary topological defects by the maskless photopatterning 
technique makes it easier to command the intrinsic molecular orders in LCEs, thus enabling programmable 3D shape transformations from 2D LCE sheets, as shown below.

\subsection{Light Actuated LCE Film with a Cone}

A mixture of $67.5 \mathrm{wt} \%$ LC monomer 4-methoxybenzoic acid 4-(6-acryloyloxyhexyloxy) phenyl ester (Synthon, Bitterfeld-Wolfen, Germany), Figure 1a, $26.0 \mathrm{wt} \%$ LC crosslinker 1,4Bis-[4-(3-acryloyloxypropyloxy) benzoyloxy]-2-methylbenzene (Wilshire INC, Princeton, NJ, United States), Figure 1b, $5.2 \mathrm{wt} \%$ azobenzene photoswitch Disperse Red 1 acrylate (DR1A, Sigma-Aldrich, St. Louis, MO, United States), Figure 1e, and $1.3 \mathrm{wt} \%$ photoinitiator (Irgacure 651, Ciba, Basel, Switzerland), Figure 1d, are dissolved in Dichloromethane (DCME, Sigma-Aldrich). Afterward, the solution is placed in the oven at $90^{\circ} \mathrm{C}$ overnight to evaporate the solvent.

A cell is constructed by using $20 \mu \mathrm{m}$ spacers to sandwich a substrate with photoaligned patterns and another substrate with homeotropic alignment. Both alignment surfaces are facing each other to induce a hybrid configuration across the sample, Figure $3 \mathrm{a}$. The prepared LCE monomer mixture is infiltrated into the chamber at $80^{\circ} \mathrm{C}$ on a hot stage. The temperature is decreased to $45^{\circ} \mathrm{C}$ at the rate of $5{ }^{\circ} \mathrm{C} / \mathrm{min}$. The solution is observed to be isotropic at $80{ }^{\circ} \mathrm{C}$ and be in the nematic phase at $45^{\circ} \mathrm{C}$. Next, the cell is irradiated by UV light with $1.4 \mathrm{~mW} / \mathrm{cm}^{2}$ intensity at a wavelength of $365 \mathrm{~nm}$ for $15 \mathrm{~min}$ to finish the polymerization. The irradiation of the UV light will not influence the photostability of the photopatterned surface, and the molecular orientations will be fixed in the LCE films after polymerization. The cell is split by a razor blade. After sonicating in a petri dish with water for $20 \mathrm{~s}$, the free-standing LCE film can be detached from the substrate by a tweezer.

Figure $3 \mathrm{~b}$ shows a topological defect of $m=+1$ with $\theta_{0}=\pi / 2$, named circular +1 defect. It is fabricated by starting the polarizer along the $x$-axis and rotating in the $\mathrm{CW}$ direction at the same speed of the segments, Figure 3c,d. Under the irradiation of a collimated LED ( $455 \mathrm{~nm}$, Thorlabs) with light intensity is $690 \mathrm{~mW}$ at its $100 \%$ beam power, the actuation of the fabricated LCE film with the bottom surface of the designed circular pattern is manifested as a cone, Figure 3e. The light actuation is enabled by the azobenzene photoswitch of DR1A, which changes the LC orders via photoisomerization. The decrease in the nematic ordering in the LCE induces contraction along the alignment direction and expansion perpendicular to it. For the sample in Figure 3e, due to the inside hybrid configuration, the perimeter contracts on the bottom surface, but the radii extend on the top surface, which can be reconciled only if a cone is formed. Moreover, this morphing is reversible, as the amplitude can be actuated by the switching state of light, Figure 3f.

\subsection{The Morphing of LCE Film with a Truncated Pyramid Shape}

In addition to a single cone, a complex $2 \times 2$ cone array is enabled by embedding the circular +1 array into the LCE film, Figure 4 . As depicted in Figure $4 a$, the director field is made of four individual circular +1 topological defects without any boundary. To achieve this pattern, the PowerPoint slides are modified to include four identical segments on every single slide, Figure $4 \mathrm{~b}$. The distance intervals of the segments along the $x$-axis and $y$-axis are the same. The polarizer and segments all start along the $x$-axis and rotate in the $\mathrm{CW}$ direction at the same speed. These four individual defects can be created at the same time after $2 \pi$ rotation of the exposed segments, Figure $4 \mathrm{c}$. Instead of four independent cones, the overall shape morphing turns out to be in a truncated square pyramid. The actuated amplitude is about $6 \mathrm{~mm}$, which is about 300 times taller than that of a flat sheet with $20 \mu \mathrm{m}$ thickness, Figure $4 \mathrm{~d}$,e. The shape of a truncated pyramid is enabled thanks to the overlapping of these topological defects. 


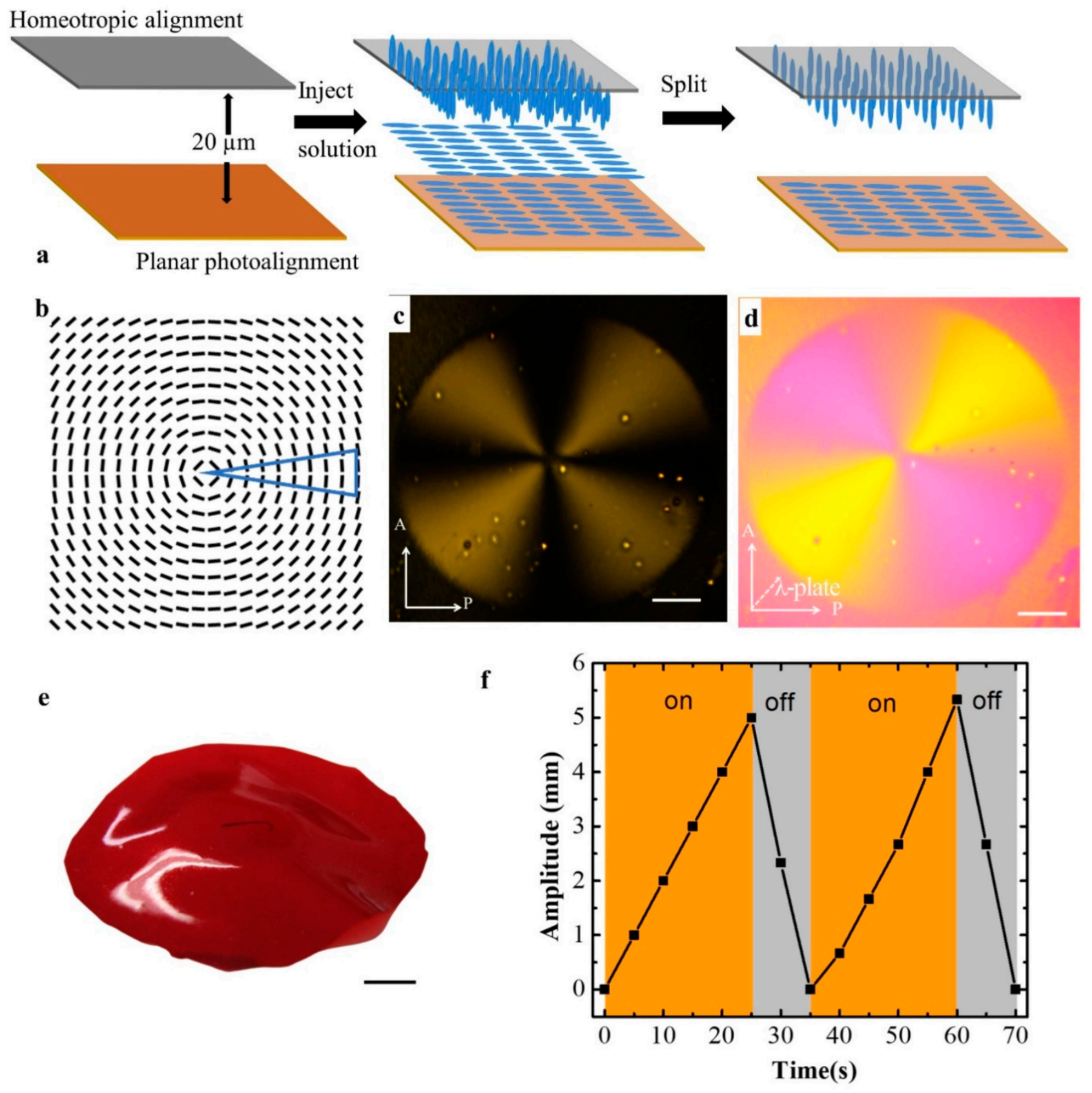

Figure 3. Fabrication of LCE film with circular +1 defect. (a) The LCE film fabrication process. (b) The director field of circular +1 defect. The blue triangle is the position of the starting segment. (c,d) The POM images without (c) and with (d) red plate. (e) A single cone is formed in LCE film with the bottom surface of a circular +1 defect. (f) The dependence of the amplitude of the LCE film on light irradiation. The scale bars are $2.5 \mathrm{~mm}$.

Previously, the shape-morphing of LCE film with a 2 D lattice of +1 topological defects was demonstrated by the White group $[14,31]$. The spatially varying director field was fabricated by shining pixel-by-pixel linearly polarized light, which is time-consuming and costly. In our work, multiple topological defects can be created at the same time, and it only takes six-minute irradiation to complete the whole pattern, which is much more efficient. Meanwhile, by adjusting the positions of the lens or replacing one of them with an objective lens in the setup, scalability of sizes from tens of micrometers to centimeters can also be enabled. 

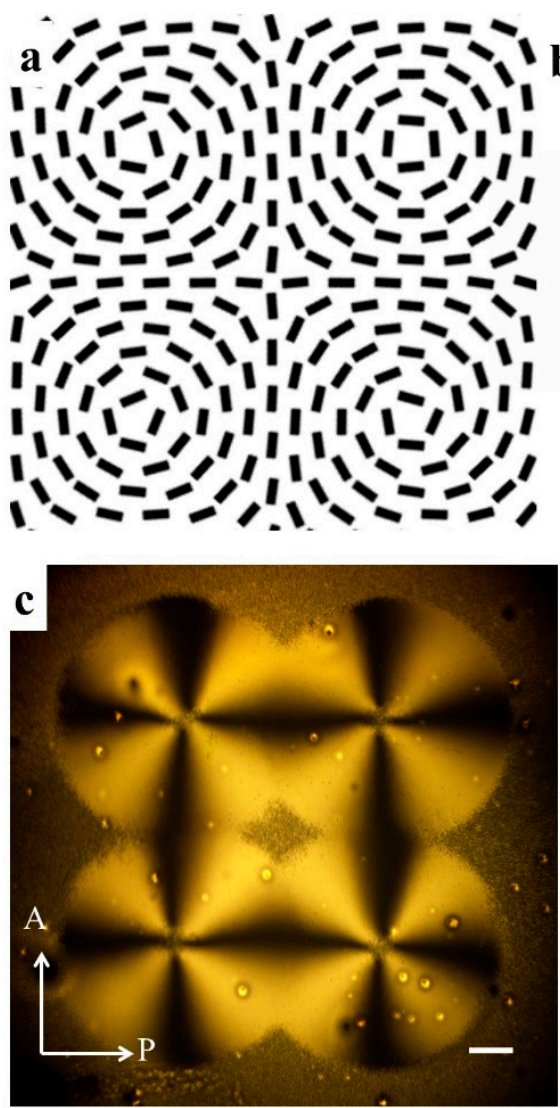
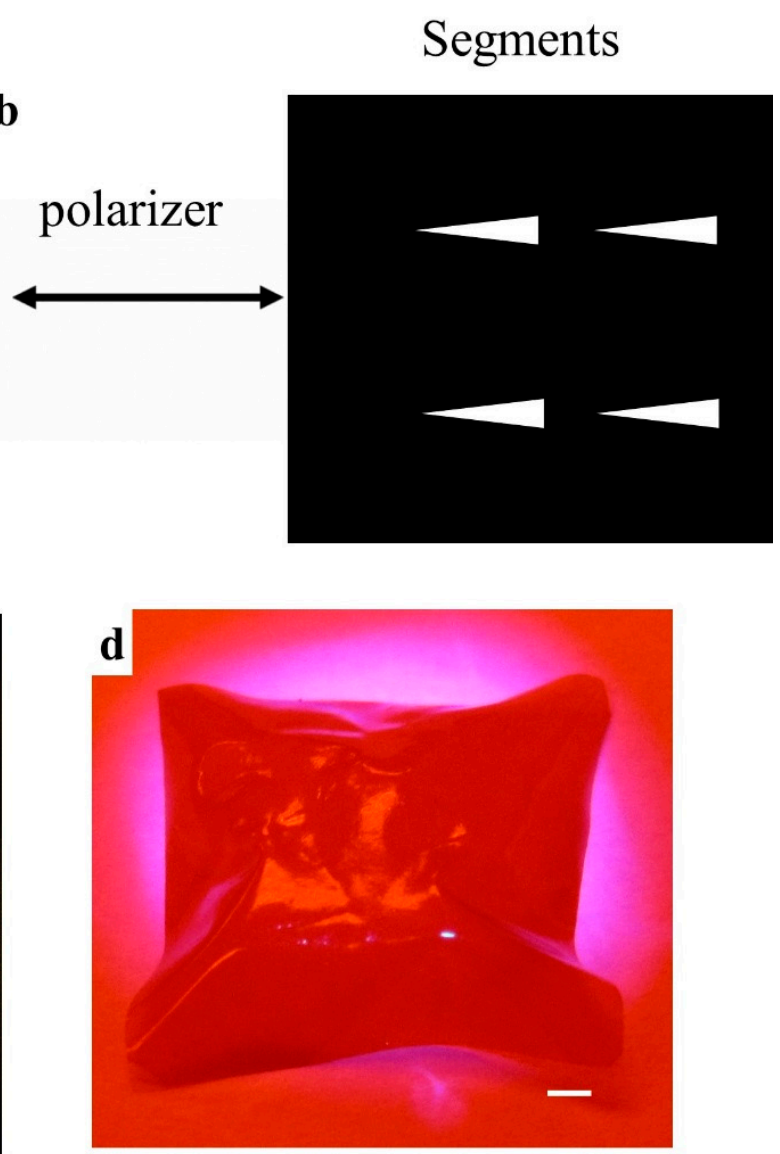

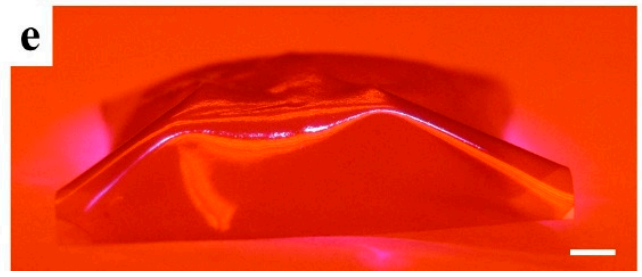

Figure 4. Shape changes of the LCE film with a 2D lattice of circular +1 defects. (a) The director filed with a $2 \mathrm{D}$ array of circular +1 defects. (b) The starting positions of the polarizer and segments. (c) The POM image of the designed pattern. (d,e) The top (d) and side (e) views of the shaping deformation of the LCE film, respectively. The scale bars are $2.5 \mathrm{~mm}$.

\subsection{Biomimetic Actuation of LCE Device}

The actuation of hierarchical assembly of LCE geometries inspired from the responsive Mimosa Pudica to the external stimuli is also demonstrated. Mimosa Pudica is a special plant that will close its leaves upon mechanical stimulation, Figure 5 a. Here, we fabricated a bioinspired LCE device of Mimosa Pudica by using the radial +1 defect with a geometric triangular shape. LCE film with radial +1 defect (shown in Figure 1g) is fabricated and cut into triangular geometries. Then four of them are hierarchically stuck to a paper clip, Figure 5b. Each of the LCE films adopts a hybrid configuration with the top surface of aligned pattern, inset of Figure 5b. Before irradiation, the biomimetic "leaves" hang down owing to a self-weight of $2 \mathrm{mg}$. Figure $5 \mathrm{c}-\mathrm{f}$ are snapshots extracted from Video S1, depicting the actuation of every pair of "leaves" under light irradiation, respectively. Due to the hybrid configuration in the LCE "leaves", the "leaves" bend upward to the "closed" state via actuation. Similar to Mimosa Pudica responding to the external stimulus, the biomimetic "leaves" only morph when the stimulus "light" is present. After "leaves" move away from the stimulus "light", their shape deformation will be reversed to the original state. The 
bending angle of the actuator is dependent on the light intensity, Figure $5 \mathrm{~g}$. The inset images in Figure $5 \mathrm{~g}$ are all taken after $5 \mathrm{~s}$ of irradiation for different intensities.
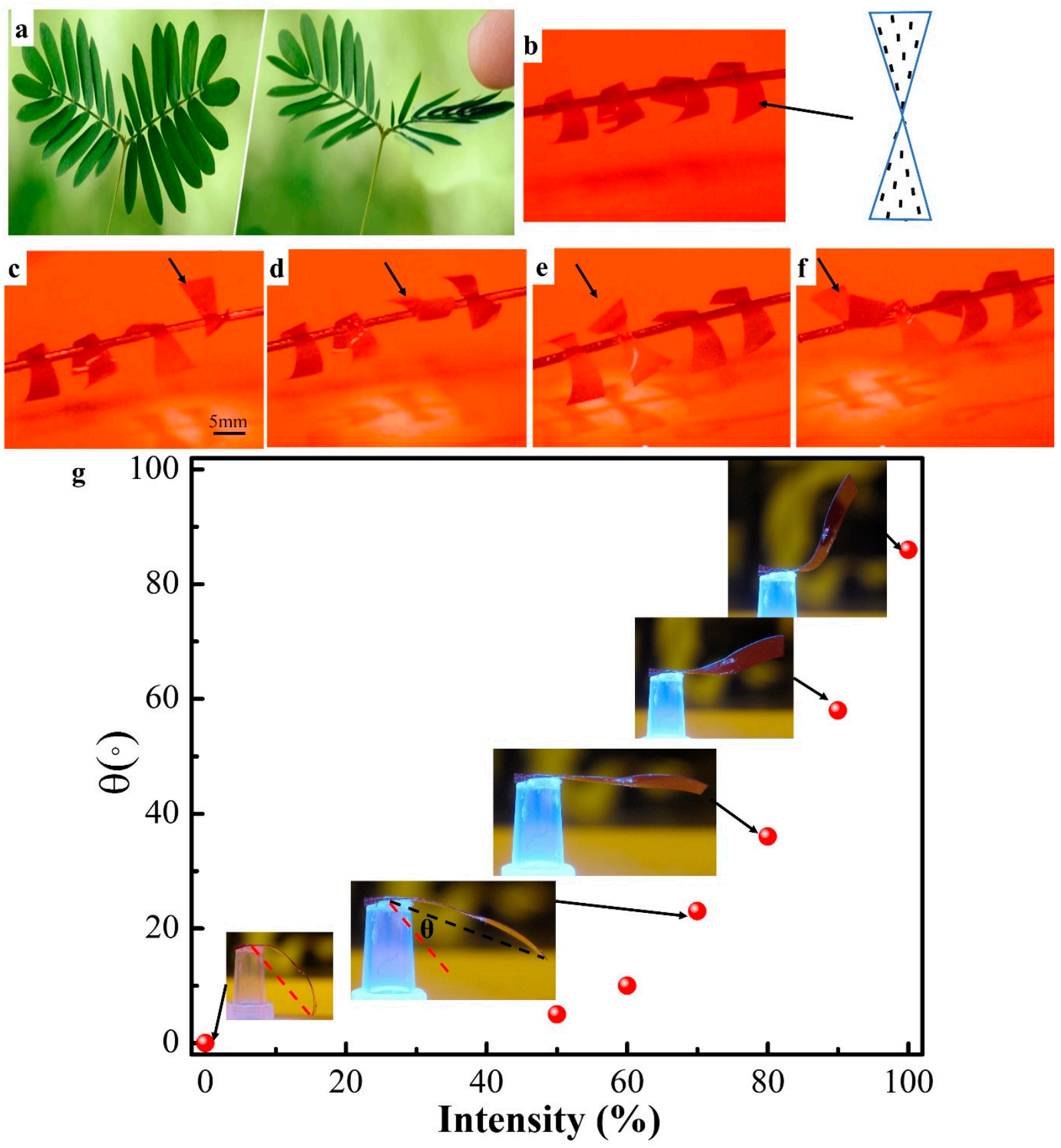

Figure 5. Bioinspired LCE Mimosa Pudica. (a) Leaves of plant Mimosa Pudica close immediately when stimulated by external forces. (b) The bioinspired LCE Mimosa Pudica before irradiation. The LCE assumes a hybrid configuration with the top surface pattern shown in the inset. (c-f) The "leaves" react to an external stimulus, "light". The black arrow indicates the leaf pairs that are under irradiation. (g) The dependence of bending angle $\theta$ on light intensity.

\section{Conclusions}

There have been extensive theoretical analyses about mechanical responses of LCE under external stimuli $[7,32-40]$. In this work, the actuation mechanism is rooted in the nematic ordering change in the LCE film. The decrease in the nematic ordering in the LCE induces contraction along the alignment direction and expansion perpendicular to it. For the LCE film combining a top surface of homeotropic alignment with a bottom surface of 
photoaligned circular pattern, the deformations in the LCE film can only be reconciled by bulging to a cone thanks to this hybrid configuration. Likewise, the shape morphology of a truncated square pyramid is also enabled based on this fundamental understanding.

In summary, we have demonstrated a cheap, efficient, and easy photopatterning technique to create spatially varying molecular orientations in LCE films by using a maskless projection display setup. A variety of individual topological defects and a 2D lattice of defect array are created by this simple method. We further integrate the topological defects into the light-fueled LCE films to achieve programmable, reversible, and remotely-actuated shape changes. The actuation of a cone, a truncated square pyramid, and a biomimetic LCE Mimosa Pudica device is realized by light irradiation. The deformation angle is dependent on the light intensity. The demonstrated programmable shape changes of LCE films can be further used in soft robotics, smart functional materials, and biomedical devices.

Supplementary Materials: The following are available online at https: / www.mdpi.com/article / 10.3390/ma14237245/s1, Video S1: Bioinspired LCE Mimosa Pudica.

Author Contributions: Conceptualization, C.P.; Data curation, J.C. and O.I.A.; Formal analysis, J.C.; Methodology, J.C. and J.J.; Supervision, C.P.; Validation, J.J. and C.P.; Writing-original draft, J.C.; Writing-review \& editing, O.I.A., J.J. and C.P. All authors have read and agreed to the published version of the manuscript.

Funding: This research received no external funding.

Institutional Review Board Statement: Not applicable.

Informed Consent Statement: Not applicable.

Data Availability Statement: The data presented in this study are available on request from the corresponding author.

Conflicts of Interest: The authors declare no conflict of interest.

\section{References}

1. Zhao, X.; Kim, J.; Cezar, C.A.; Huebsch, N.; Lee, K.; Bouhadir, K.; Mooney, D.J. Active scaffolds for on-demand drug and cell delivery. Proc. Natl. Acad. Sci. USA 2011, 108, 67-72. [CrossRef] [PubMed]

2. Liu, Z.; Calvert, P. Multilayer Hydrogels as Muscle-Like Actuators. Adv. Mater. 2000, 12, 288-291. [CrossRef]

3. Zhang, Y.S.; Khademhosseini, A. Advances in engineering hydrogels. Science 2017, 356, 3627. [CrossRef] [PubMed]

4. Ma, M.; Guo, L.; Anderson, D.G.; Langer, R. Bio-Inspired Polymer Composite Actuator and Generator Driven by Water Gradients. Science 2013, 339, 186. [CrossRef] [PubMed]

5. Zarek, M.; Layani, M.; Cooperstein, I.; Sachyani, E.; Cohn, D.; Magdassi, S. 3D Printing of Shape Memory Polymers for Flexible Electronic Devices. Adv. Mater. 2016, 28, 4449. [CrossRef]

6. Webber, M.J.; Appel, E.A.; Meijer, E.W.; Langer, R. Supramolecular biomaterials. Nat. Mater. 2016, 15, 13. [CrossRef]

7. Warner, M.; Terentjev, E.M. Liquid Crystal Elastomers; Oxford University Press: New York, NY, USA, 2007 ; Volume 120.

8. Gelebart, A.A.H.; Mulder, D.J.; Varga, M.; Konya, A.; Vantomme, G.; Meijer, E.B.; Selinger, R.; Broer, D.J. Making waves in a photoactive polymer film. Nature 2017, 546, 632. [CrossRef] [PubMed]

9. McConney, M.E.; Martinez, A.; Tondiglia, V.P.; Lee, K.M.; Langley, D.; Smalyukh, I.I.; White, T.J. Topography from Topology: Photoinduced Surface Features Generated in Liquid Crystal Polymer Networks. Adv. Mater. 2013, 25, 5880. [CrossRef] [PubMed]

10. Wani, O.M.; Verpaalen, R.; Zeng, H.; Priimagi, A.; Schenning, A.P.H.J. An Artificial Nocturnal Flower via Humidity-Gated Photoactuation in Liquid Crystal Networks. Adv. Mater. 2019, 31, 1805985. [CrossRef]

11. Zhu, C.; Lu, Y.; Jiang, L.; Yu, Y. Liquid Crystal Soft Actuators and Robots toward Mixed Reality. Adv. Funct. Mater. 2021, 1, 2009835. [CrossRef]

12. Xiao, Y.-Y.; Jiang, Z.-C.; Zhao, Y. Liquid Crystal Polymer-Based Soft Robots. Adv. Intell. Syst. 2020, 2, 2000148. [CrossRef]

13. Tan, L.; Davis, A.C.; Cappelleri, D.J. Smart Polymers for Microscale Machines. Adv. Funct. Mater. 2021, 31, 2007125. [CrossRef]

14. Ware, T.H.; McConney, M.E.; Wie, J.J.; Tondiglia, V.P.; White, T.J. Voxelated liquid crystal elastomers. Science $2015,347,982$. [CrossRef] [PubMed]

15. Aharoni, H.; Xia, Y.; Zhang, X.; Kamien, R.D.; Yang, S. Universal inverse design of surfaces with thin nematic elastomer sheets. Proc. Natl. Acad. Sci. USA 2018, 115, 7206. [CrossRef] [PubMed]

16. Herbert, K.M.; Fowler, H.E.; McCracken, J.M.; Schlafmann, K.R.; Koch, J.A.; White, T.J. Synthesis and alignment of liquid crystalline elastomers. Nat. Rev. Mater. 2021, 1-16. [CrossRef]

17. White, T.J.; Broer, D.J. Programmable and Adaptive Mechanics with Liquid Crystal Polymer Networks and Elastomers. Nat. Mater. 2015, 14, 1087. [CrossRef] [PubMed] 
18. Murray, B.S.; Pelcovits, R.A.; Rosenblatt, C. Creating arbitrary arrays of two-dimensional topological defects. Phys. Rev. E 2014, 90, 052501. [CrossRef] [PubMed]

19. Harkai, S.; Murray, B.S.; Rosenblatt, C.; Kralj, S. Electric field driven reconfigurable multistable topological defect patterns. Phys. Rev. Res. 2020, 2, 013176. [CrossRef] [PubMed]

20. Xia, Y.; Cedillo-Servin, G.; Kamien, R.D.; Yang, S. Guided Folding of Nematic Liquid Crystal Elastomer Sheets into 3D via Patterned 1D Microchannels. Adv. Mater. 2016, 28, 9637. [CrossRef]

21. Guo, Y.; Shahsavan, H.; Davidson, Z.; Sitti, M. Precise Control of Lyotropic Chromonic Liquid Crystals Alignment through Surface Topography. ACS Appl. Mater. Interfaces 2019, 11, 36110. [CrossRef]

22. del Pozo, M.; Sol, J.A.H.P.; Schenning, A.P.H.J.; Debije, M.G. 4D Printing of Liquid Crystals: What's Right for Me? Adv. Mater. 2021, 2104390. [CrossRef] [PubMed]

23. Yaroshchuk, O.; Reznikov, Y. Photoalignment of liquid crystals: Basics and current trends. J. Mater. Chem. 2012, 22, 286. [CrossRef]

24. Peng, C.; Guo, Y.; Turiv, T.; Jiang, M.; Wei, Q.; Lavrentovich, O.D. Patterning of Lyotropic Chromonic Liquid Crystals by Photoalignment with Photonic Metamasks. Adv. Mater. 2017, 29, 1606112. [CrossRef] [PubMed]

25. Culbreath, C.; Glazar, N.; Yokoyama, H. Note: Automated maskless micro-multidomain photoalignment. Rev. Sci. Instrum. 2011, 82, 126107. [CrossRef] [PubMed]

26. Guo, Y.; Jiang, M.; Peng, C.; Sun, K.; Yaroshchuk, O.; Lavrentovich, O.; Wei, Q.H. High-Resolution and High-Throughput Plasmonic Photopatterning of Complex Molecular Orientations in Liquid Crystals. Adv. Mater. 2016, 28, 2353. [CrossRef] [PubMed]

27. Peng, C.; Turiv, T.; Guo, Y.; Wei, Q.-H.; Lavrentovich, O.D. Command of Active Matter by Topological Defects and Patterns. Science 2016, 354, 882. [CrossRef]

28. Nersisyan, S.; Tabiryan, N.; Steeves, D.M.; Kimball, B.R. Fabrication of liquid crystal polymer axial waveplates for UV-IR wavelengths. Opt. Express 2009, 17, 11926. [CrossRef]

29. Slussarenko, S.; Murauski, A.; Du, T.; Chigrinov, V.; Marrucci, L.; Santamato, E. Tunable liquid crystal q-plates with arbitrary topological charge. Opt. Express 2011, 19, 4085. [CrossRef] [PubMed]

30. De Haan, L.T.; Sánchez-Somolinos, C.; Bastiaansen, C.M.W.; Schenning, A.P.H.J.; Broer, D.J. Engineering of complex order and the macroscopic deformation of liquid crystal polymer networks. Angew. Chem. 2012, 124, 12637. [CrossRef]

31. Ahn, S.K.; Ware, T.H.; Lee, K.M.; Tondiglia, V.P.; White, T.J. Photoinduced Topographical Feature Development in Blueprinted Azobenzene-Functionalized Liquid Crystalline Elastomers. Adv. Funct. Mater. 2016, 26, 5819. [CrossRef]

32. Modes, C.D.; Bhattacharya, K.; Warner, M. Disclination-mediated thermo-optical response in nematic glass sheets. Phys. Rev. E 2010, 81, 060701. [CrossRef] [PubMed]

33. Modes, C.D.; Bhattacharya, K.; Warner, M. Gaussian curvature from flat elastica sheets. Proc. R. Soc. A Math. Phys. Eng. Sci. 2011, 467, 1121. [CrossRef]

34. Modes, C.D.; Warner, M. Blueprinting nematic glass: Systematically constructing and combining active points of curvature for emergent morphology. Phys. Rev. E 2011, 84, 021711. [CrossRef] [PubMed]

35. Kowalski, B.A.; Mostajeran, C.; Godman, N.P.; Warner, M.; White, T.J. Curvature by design and on demand in liquid crystal elastomers. Phys. Rev. E 2018, 97, 012504. [CrossRef] [PubMed]

36. Gimenez-Pinto, V.; Ye, F.; Mbanga, B.; Selinger, J.; Selinger, R. Modeling out-of-plane actuation in thin-film nematic polymer networks: From chiral ribbons to auto-origami boxes via twist and topology. Sci. Rep. 2017, 7, 45370. [CrossRef]

37. Lavrentovich, O.D. Prepatterned liquid crystal elastomers as a step toward artificial morphogenesis. Proc. Natl. Acad. Sci. USA 2018, 115, 7171. [CrossRef]

38. Pei, Z.; Yang, Y.; Chen, Q.; Terentjev, E.; Wei, Y.; Ji, Y. Mouldable liquid-crystalline elastomer actuators with exchangeable covalent bonds. Nat. Mater. 2013, 13, 36. [CrossRef] [PubMed]

39. Mihai, L.A.; Wang, H.; Guilleminot, J.; Goriely, A. Nematic liquid crystalline elastomers are aeolotropic materials. Proc. R. Soc. A Math. Phys. Eng. Sci. 2021, 477, 20210259. [CrossRef]

40. Duffy, D.; Biggins, J.S. Defective nematogenesis: Gauss curvature in programmable shape-responsive sheets with topological defects. Soft Matter. 2020, 16, 10935. [CrossRef] [PubMed] 